\title{
Linear motion correction in three dimensions applied to dynamic gadolinium enhanced breast imaging
}

\author{
Sumati Krishnan, Thomas L. Chenevert, ${ }^{\text {a) }}$ Mark A. Helvie, and Frank L. Londy \\ 1500 E. Medical Center Drive, Department of Radiology, University of Michigan Hospitals, \\ Ann Arbor, Michigan 48109
}

(Received 8 May 1998; accepted for publication 24 February 1999)

\begin{abstract}
Quantitative analysis of dynamic gadolinium-DTPA (diethylenetriamine pentaacetic acid) enhanced magnetic resonance imaging (MRI) is emerging as a highly sensitive tool for detecting malignant breast tissue. Three-dimensional rapid imaging techniques, such as keyhole MRI, yield high temporal sampling rates to accurately track contrast enhancement and washout in lesions over the course of multiple volume acquisitions. Patient motion during the dynamic acquisitions is a limiting factor that degrades the image quality, particularly of subsequent subtraction images used to identify and quantitatively evaluate regions suggestive of malignancy. Keyhole imaging is particularly sensitive to motion since datasets acquired over an extended period are combined in $k$-space. In this study, motion is modeled as set of translations in each of the three orthogonal dimensions. The specific objective of the study is to develop and implement an algorithm to correct the consequent phase shifts in $k$-space data prior to offline keyhole reconstruction three-dimensional (3D) volume breast MR acquisitions. (C) 1999 American Association of Physicists in Medicine.
\end{abstract} [S0094-2405(99)01005-6]

Key words: breast, neoplasms, keyhole, linear motion, post-processing algorithms

\section{INTRODUCTION}

Contrast enhanced magnetic resonance imaging (MRI) is a developing technique for characterization of breast lesions. ${ }^{1-8}$ This method is based on the tendency of lesions to exhibit enhancement after administration of gadoliniumbased contrast media. Furthermore, malignant tissues typically exhibit rapid enhancement due to anomalous vascularity, in which the combination of increased capillary density and permeability results in rapid enhancement, and occasionally washout, of the contrast agent in a dynamic contrast MRI study. ${ }^{9-12}$ Benign tissues, on the other hand, tend to have a slower enhancement rate. ${ }^{5}$ Some benign tissues, such as fibroadenomas, have been observed to exhibit rapid enhancement, ${ }^{13}$ thereby reducing the specificity of dynamic contrast enhanced MRI of the breast. Nevertheless, analysis of the temporal enhancement patterns is considered an emerging tool that can be used for detection and differentiation of various breast lesions. For this purpose, twodimensional multisection breast MR imaging has been performed with an associated temporal resolution of $\sim 1$ min. $^{5}$ Even better temporal resolution (15 s) has been achieved by imaging one or a few pre-selected slices. ${ }^{7}$ Realistically, selection of a few slices based on findings at $\mathrm{x}$-ray mammography, ultrasonography, or physical examination can be difficult and is useful only in characterizing known lesions. Correlation of these findings to a few MRI imaging slices is difficult due to the variable deformation of the breast. Also, since certain lesion are not easily identified in the absence of contrast agents, a precontrast MR image by itself could prove inadequate for prospective selection of appropriate imaging sections. Thus for dynamic MR breast imaging to be a useful screening tool, techniques that provide $3 \mathrm{D}$ volume images of one or both breasts while sampling contrast enhancement information at high temporal resolution, are desired.

Three-dimensional dynamic MRI consists of acquiring serial 3D volume images over a finite time period that encompasses contrast enhancement. The time per acquisition is a function of the imaging parameters, such as matrix size, number of excitations (NEX), and the type of acquisition sequence used. ${ }^{14}$ Three-dimensional volume imaging with fat suppression using magnetization transfer contrast has been performed to yield images of good anatomic quality, i.e., high spatial resolution, but with acquisition times ranging to several minutes. ${ }^{15}$ Rapidly enhancing tissues have been observed to reach peak enhancement on the order of tens of seconds. To be sensitive to the most rapidly enhancing tissues high sampling rates are required which is achieved, in part, by using fast imaging techniques, such as a 3D fast rf-spoiled gradient recalled echo (3D SPGR) sequence used in this study. Despite improvements in the speed of the imaging pulse sequence, there remains a tradeoff between high spatial resolution and high temporal sampling rates, which relates to the objective of breast lesion detection and characterization. One approach to address this tradeoff has been to subsample $k$-space using a tailored traversal pattern, while maintaining a high dynamic temporal sampling rate. The resulting spatial resolution usually depends upon the extent of $k$-space traversal and the reconstruction algorithm. Examples include reduced-encoding imaging using generalized reconstruction (RIGR),${ }^{16}$ use of projection reconstruction trajectories, ${ }^{17}$ and dynamic spiral breast imaging. ${ }^{18}$ A particular technique, used for data acquisition in this study, that similarly mitigates the spatial- 
temporal tradeoff is the "keyhole" MRI technique described in the following section.

The keyhole technique involves the acquisition of one or more reference high-resolution datasets followed by a dynamic series of datasets having a limited extent in $k$-space. ${ }^{19}$ The reduced matrix acquisition usually samples only the central portion of $k$-space, which is then spliced with peripheral $k$-space data from a full matrix reference dataset acquired initially. In keyhole acquisitions it is assumed that the significant dynamic events (such as amplitude changes in breast lesions), are tracked by acquiring the central $k$-space lines. The adequacy of tracking changes depend on the spatial features of the enhancing object. It is an inherent limitation of keyhole imaging that features exhibiting temporal enhancement will be blurred by an amount dependent on the keyhole matrix size and the spatial dimensions of the object. Fine structures with a higher peripheral spatial frequency content will be blurred by a greater amount. ${ }^{20}$ However, the spatial detail of static, nonenhancing, background structures is restored by splicing together the high spatial frequencies from the reference dataset. This serves to provide a highresolution anatomic context for evaluating the dynamically changing lesions. $^{21}$

A significant source of error related to keyhole imaging will be due to breast motion over the total acquisition period. This will manifest as phase differences between the reference and dynamic datasets. For contrast enhanced MRI, quantitative analysis is commonly performed on subtraction images that emphasize temporal changes. Keyhole reconstruction of the phase-deviant datasets will result in substantial edge artifacts and blurring in the subsequent subtraction images used for quantitative analysis. ${ }^{22}$ A number of motion models and corresponding post-processing techniques have been proposed to reduce motion artifact for two-dimensional Fourier transform imaging. ${ }^{23-27}$ The purpose of this paper is to outline a 3D model for motion during the rapid dynamic acquisition and describe a method of estimation and correction for the phase artifacts introduced by motion. The motion correction algorithm was initially verified by computer simulation. It was tested on a phantom experiment dataset and applied to clinical breast studies.

\section{MATERIALS AND METHODS}

\section{Motion correction}

Dynamic breast imaging data was acquired using a dual phased array coil, which permitted processing of data from the right and left coils separately. Thus motion correction was applied individually to each breast. The motion model was based on a consideration of the patient configuration and imaging rates. With some mild compression most patients were reasonably well constrained within the breast coil. The 3D SPGR imaging sequence acquired a single 3D volume once every $\sim 10 \mathrm{~s}$. Since the patients were cautioned to hold still, the most likely causes of motion were gradual positional shifts that evolved on a time scale longer than the dynamic temporal sampling rate. Thus, we assume negligible intrakeyhole motion and consider a mean position over each dynamic volume acquisition. Based on visual inspection of the subtraction edge artifacts observed, we further limit our analysis to simple translations in each of the three orthogonal dimensions. Our primary focus, therefore, was to detect and correct simple displacements that occurred over the duration of the overall dynamic acquisition on a per keyhole basis. Thus, the model assumed that after the reference acquisition and between each subsequent dynamic acquisition, the individual breasts were allowed to move independently as rigid bodies undergoing only translational motion.

It is known that a simple displacement in space introduces a corresponding phase shift in the spatial frequency signal while the magnitude of the data remains unchanged. ${ }^{28}$ Thus there is a phase difference between the reference dataset and the dynamic dataset acquired after the object has been displaced. Let $S\left(k_{x}, k_{y}, k_{z}\right)$ represent the reference dataset in the spatial frequency domain, given by

$$
S\left(k_{x}, k_{y}, k_{z}\right)=\int \rho(x, y, z)^{e\left(i\left(k_{x} x+k_{y} y+k_{z} z\right)\right)} d x d y d z .
$$

Now, let the object, $\rho(x, y, z)$, be displaced by $\Delta x, \Delta y, \Delta z$ at a given time point. The new position of the object can be described as a convolution with a displaced delta function

$$
\rho^{\prime}(x, y, z)=\rho(x, y, z) \otimes \delta(x-\Delta x, y-\Delta y, z-\Delta z) .
$$

Consequently, the $k$-space data from the object is now given by

$$
S^{\prime}\left(k_{x}, k_{y}, k_{z}\right)=S\left(k_{x}, k_{y}, k_{z}\right)^{e\left(-i\left(k_{x} \Delta x+k_{y} \Delta y+k_{z} \Delta z\right)\right)} .
$$

Thus the net phase shift due to $3 \mathrm{D}$ translation is given as

$$
\phi\left(k_{x}, k_{y}, k_{z}\right)=k_{x} \Delta x+k_{y} \Delta y+k_{z} \Delta z .
$$

In keeping with our motion model, the objective of the motion correction algorithm was to estimate this linear phase shift of the entire keyhole $k$-space block relative to the reference data block per spatial frequency axis, and apply a phase correction to each dataset prior to keyhole reconstruction. The approach was to compute an averaged linear phase roll along each individual $k$-space axis, [Eq. (5)] which is in effect a projection of mean phase difference over the 3D dataset, onto that particular axis.

To implement the algorithm, first a phase difference matrix was generated between a central $(32 \times 32 \times 32)$ kernel extracted from the reference dataset and each dynamic time point. The phase difference was estimated on reduced matrix spatial frequency datasets, because it cannot be reliably computed in the low amplitude, peripheral $k$-space, regions of the signal. In these regions the noise amplitude is comparable to that of the signal and hence the phase could fluctuate randomly, obscuring the effect of linear motion. The average phase difference along each spatial frequency axis was computed as follows:

$$
\begin{aligned}
\phi\left(k_{x}\right)= & \frac{1}{\left(n_{y} \cdot n_{z}\right)} \sum_{k_{y}} \sum_{k_{z}} \cdot \phi\left(k_{x}, k_{y}, k_{z}\right) \\
& + \text { constant term, }
\end{aligned}
$$




$$
\begin{aligned}
\phi\left(k_{y}\right)= & \frac{1}{\left(n_{x} \cdot n_{z}\right)} \sum_{k_{x}} \sum_{k_{z}} \cdot \phi\left(k_{x}, k_{y}, k_{z}\right) \\
& + \text { constant term, } \\
\phi\left(k_{z}\right)= & \frac{1}{\left(n_{x} \cdot n_{y}\right)} \sum_{k_{x}} \sum_{k_{y}} \cdot \phi\left(k_{x}, k_{y}, k_{z}\right) \\
& + \text { constant term, }
\end{aligned}
$$

where, $n_{x}, n_{y}, n_{z}$, is the matrix size and, $\phi\left(k_{x}\right), \phi\left(k_{y}\right)$, $\phi\left(k_{y}\right)$, are the averaged phase deviations per $k$-space axis.

Thus we obtain a phase difference function per $k$-space axis which is a measure of the averaged phase difference between the reference and dynamic datasets, due to displacement in the corresponding spatial axis during a particular dynamic timepoint. For example, in Fig. 1(a) the coronal plane image shows a noticeable edge artifact. Figure 1(b) in turn, shows a net averaged linear phase roll detected along the $k_{x}$ and $k_{z}$ dimensions and negligible phase deviations in $k_{y}$. This implies linear displacement along the $x$ and $z$ dimensions. Thus the phase function is an estimate of the translation induced phase shift. The linear phase function could contained wrapped phase depending on the extent of motion along a particular spatial axis. Thus, an unwrapping algorithm was applied to each function so that it increased or decreased monotonically. These averaged phase deviation functions were submitted to a linear least squares estimation algorithm from which a representative slope per spatial frequency axis was computed. This slope was used to correct the phase deviant dataset by simply applying an inverse linear phase ramp such that

$$
\phi_{\text {corr }}\left(k_{x}, k_{y}, k_{z}\right)=k_{x} s_{x}+k_{y} s_{y}+k_{z} s_{z}
$$

where, $s_{x}, s_{y}$, and $s_{z}$ were the fitted slopes in $k_{x}, k_{y}$, and $k_{z}$.

The constant phase shift term between the reference and the slope-corrected datasets was also determined and incorporated in the phase correction algorithm. In the case of very large displacements $\left[>\frac{1}{2}\right.$ (FOV) field of view] along each axis, the phase difference function could be wrapped twice. The unwrapping technique used would not be able to account for this in a single stage and hence the correction algorithm was applied to the data in two consecutive stages.

\section{ANALYSIS OF AMPLITUDE MODULATION EFFECTS}

The amplitude modulation of the data that is associated with uptake of Gd-DTPA could theoretically, be misinterpreted as a phase shift. Computer simulations were used to further analyze this effect. We investigated the effect of signal enhancement by considering signal increases starting from 100 to a maximum of 500 percent. Two simplified anatomic configurations of breast fat and lesion were considered. First, we varied the percentage of simulated lesion area to breast fat area from $1 \%-60 \%$. We increased lesion size relative to the size of the background fat while changing the
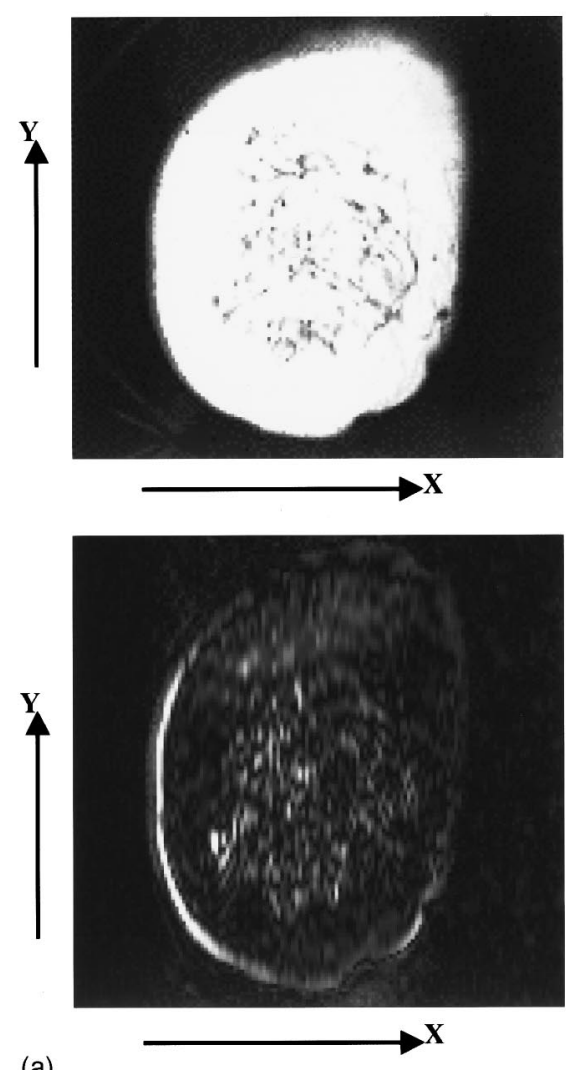

(a)

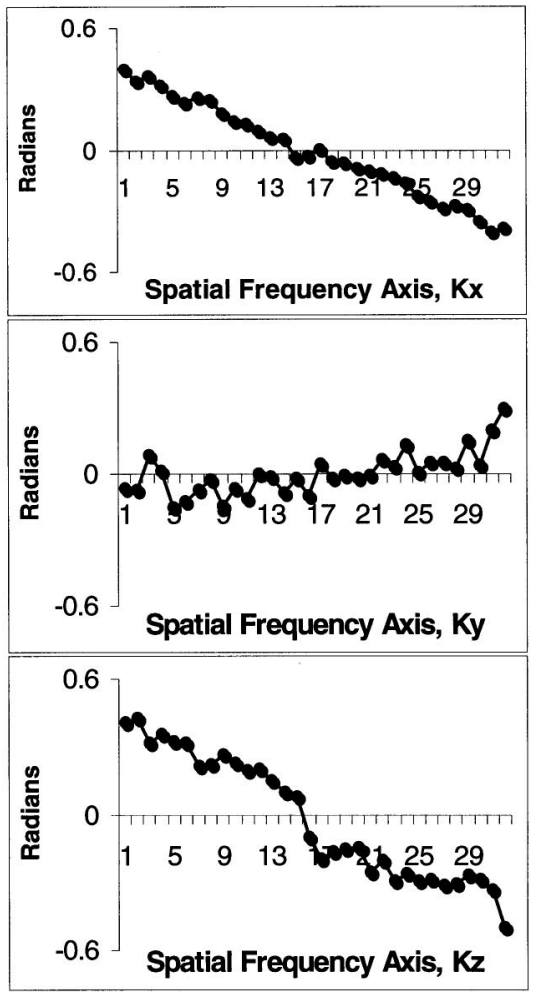

(b)

FIG. 1. (a) Reconstructed image of single (right) breast after separating dual phased array data. Bottom image shows representative subtraction artifact on coronal slice of dynamic timepoint 14. (b) Averaged phase deviations per spatial frequency axis for dynamic timepoint shown in (a), relative to the reference dataset. 
percent signal enhancement within the lesion. The phase difference between each enhanced and the original unenhanced dataset was computed. Second, we varied the displacement between the axes of the simulated breast fat and lesion from 0 to $5 \mathrm{~cm}$. We displaced breast fat relative to a fixed lesion. Again, for each axial displacement configuration the percent signal enhancement was varied and the phase difference between the enhanced and unenhanced datasets was computed.

\section{MR imaging}

The breast studies were performed on a $1.5 T$ system (General Electric Medical Systems, Milwaukee, Wisconsin), using a dedicated breast dual phased array coil. For the clinical data, $T 1$ and $T 2$ weighted axial scans were performed initially. A pre-contrast coronal reference dataset was acquired using the locally developed $3 \mathrm{D}$ fast rf-spoiled gradient-recalled echo (3D SPGR) sequence, 40 degree flip angle. Imaging parameters were a TR/TE of $\sim 13 / 5 \mathrm{~ms}$, acquisition matrix, $256 \times 128 \times 32$ with $3-5 \mathrm{~mm}$ thick sections and four excitations. The field of view (FOV) ranged from 28 to $36 \mathrm{~cm}$ (typically $32 \mathrm{~cm}$ ). The dynamic contrastenhanced series consisted of 20 serial 3D volumes acquisitions with a reduced matrix of $256 \times 32 \times 32$ and single excitation, followed by one full matrix dataset. The dynamic segment spanned $\sim 5$ min during which a bolus injection of Gd-DTPA was administered within the first $30-45 \mathrm{~s}$ of the scan, at a dose of $0.1 \mathrm{mmol}$ per kilogram body weight. Each dynamic 3D dataset was acquired at $12.3 \mathrm{~s}$ intervals, which is equivalent to the overall temporal resolution. Finally a series of post-contrast coronal 3D SPGR and an axial $T 1$ weighted scan were performed.

Keyhole reconstruction of the data was performed offline using custom software programs developed in AVS (Advanced Visual Systems Inc., Waltham MA). The low spatial frequencies from each dynamic dataset were spliced into the reference dataset to create the corresponding full matrix dynamic dataset, for subsequent 3D Fourier transform reconstruction. Cinegraphic loops of reconstructed anatomic and subtraction images were reviewed on the workstation. A precontrast time point was used as the subtraction mask.

\section{Phantom studies}

The motion-correction algorithm was calibrated experimentally. Displacements were introduced in each of the three spatial axes using a lever arm rigidly attached to the experimental phantom located in the breast coil. Motion was induced by translating the lever arm over a calibration scale such that the phantom was displaced by an exact amount, in increments of $2 \mathrm{~mm}$. The maximum displacements introduced were $2 \mathrm{~cm}$ along the $x$ and $y$ axes, and $1 \mathrm{~cm}$ along the $z$ axis.

The 3D dynamic simulation experiment was performed on a breast-mimicking phantom, on the 1.5T GE system. Mineral oil was used to simulate breast fat and water to simulate breast parenchyma in each of the phantom compartments. Motion was induced in only one breast phantom compartment by raising and laterally displacing a lever attached

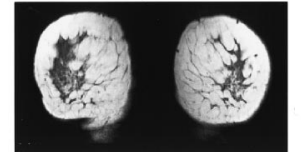

(a)

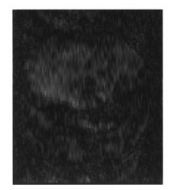

(c)

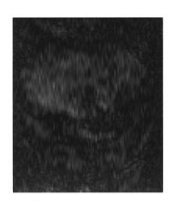

(d)

24.6”

73.8”
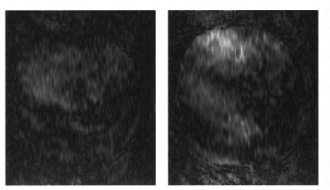

$\rightarrow$ Time

$123 "$

172.2"

221.4"

FIG. 2. Coronal slice of 3D volume acquisition (a). Anatomic image of uncorrected coronal slice. (b) Subtraction image of the same slice with clearly visible subtraction edge artifact. (c) Time series of edge artifact in the same slice prior to motion correction, $49.2 \mathrm{~s}$ intervals. (d) Time series of edge artifact in the same slice after motion correction, $49.2 \mathrm{~s}$ intervals.

to the phantom. Motions were designed to simulate the slow displacement commonly observed over the duration of the scan. Uptake of contrast in a lesion was simulated by injection of $10 \mathrm{cc}$ of $2 \mathrm{mmol}$ Gd-DTPA into $5 \mathrm{cc}$ vials located in each breast phantom compartment.

\section{Patient studies}

The clinical data included 64 breast patients who were scanned as part of an ongoing research project. Study population consisted of patients with abnormal mammograms or ultrasonically detected suspicious masses who were referred for MR scans. Patients were scanned prone with their breast suspended in the coil. Mild compression was applied to the breasts but no rigid immobilization techniques were employed. The standard clinical procedure was to caution the patients to hold still during and in between scans.

\section{Quantitative assessment}

The effectiveness of the motion correction algorithm was quantitatively assessed by comparing subtraction edge artifact prior to and after motion correction. A single measure that summarized edge artifact was devised and computed by the following procedure. For each of the 64 breast cases a representative mid-breast slice was chosen. Based on the subtraction images, a single region of interest (ROI) encompassing all of the noticeable motion artifact induced edge was defined (Fig. 2). This ROI was used as a mask over the entire sequence of dynamic timepoints and a mean ROI intensity per time point was computed, for the corrected and uncorrected datasets at the selected slice of interest. Next a baseline value equal to the mean ROI intensity from the second time point in a series was subtracted off from all 
other time points, providing the desired estimate of subtraction edge artifact. This temporal series of mean artifact ROI's was further approximated to a single mean and peak artifact ROI over the entire dynamic acquisition. Similar mean ROI's intensities were calculated for a region in each dynamic time point that corresponded to the background noise signal, for both corrected and uncorrected datasets. In order to compare the edge artifact correction across patients, the artifact ROI intensities were normalized by the mean background noise ROI intensity.

\section{RESULTS}

The calibration experiment for the motion correction algorithm yielded the following results. The correlation coefficient between the induced and detected displacements was 0.999 for the $x, y$, and $z$ dimensions. Thus, there was a close overall agreement between the induced and detected displacement along each of the spatial axes. A minimum displacement of $2 \mathrm{~mm}$ was induced and detected along each spatial axis. The theoretical limit to the maximum displacement that could be corrected for, is equivalent to a half field of view along that particular spatial axis.

The phase slopes in $k_{x}, k_{y}$, and $k_{z}$ measured in the $3 \mathrm{D}$ dynamic experimental data, corresponded to a maximum displacement of $\sim 6 \mathrm{~mm}$ along the anterior-posterior direction $(\mathrm{A} / \mathrm{P}), 2 \mathrm{~mm}$ along the right-left $(\mathrm{R} / \mathrm{L})$ direction and negligible motion along the superior-inferior (S/I) direction. Figure 3 demonstrates the effect of motion correction on the phantom. A marked reduction in motion-induced blurring and ghosting was observed on the subtracted and nonsubtracted images. An overall improvement in the edge artifact at breast boundaries as well as good background suppression was achieved for the motion-corrected subtraction images. It is interesting to note that after motion correction the vial on the right [Fig. 3(d)] is no longer visible. This is because the vial is truly outside the level of the selected slice in the 3D volume. It is visible on the uncorrected image due to subtraction artifact. After motion correction the slice is reregistered to the appropriate cross sectional level.

There was no significant difference in the mean ROI computed for each vial due to motion correction compared to the dataset without motion correction. This result was expected since the size of the simulated lesion was fairly large and therefore keyhole imaging could accurately track dynamic changes.

The simulation investigating the effect of contrast modulation on phase estimation showed enhancement effects to be negligible. We found that, as long as the high signal unenhancing breast fat, was larger in area or mostly coaxial with the enhancing lesion, the phase deviations were very small for all conditions of enhancement within the lesion. The computed phase errors were negligible for lesion as large as $60 \%$ of breast area with axial separations up to $5 \mathrm{~cm}$. This was validated by the phantom experiment where the lesionsimulating vial was small compared to the breast fat compartment and located coaxially. There was no significant difference between the mean ROI in the signal enhancing vial

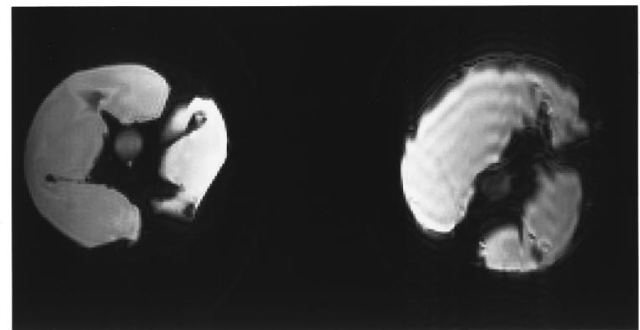

(a)

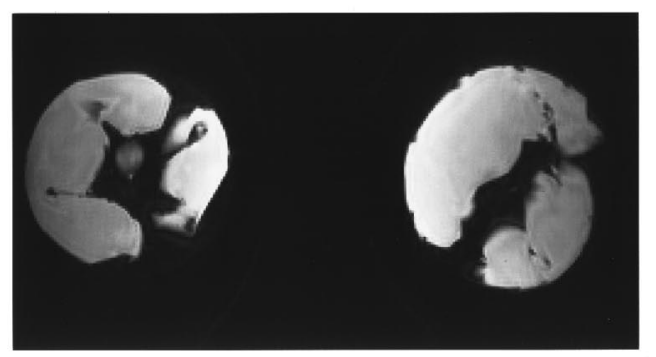

(b)

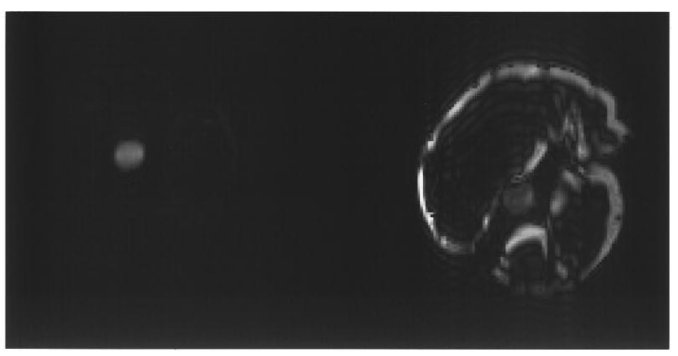

(c)

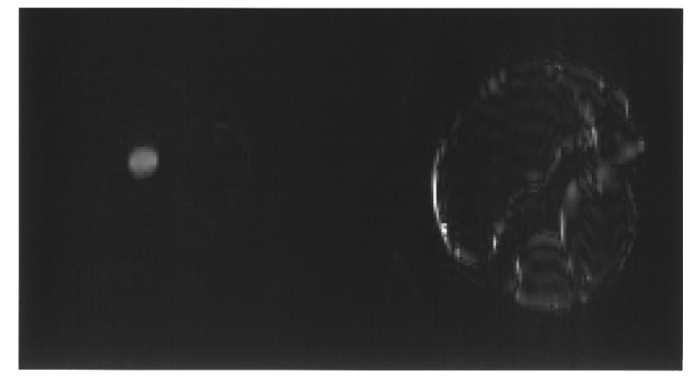

(d)

FIG. 3. Phantom experiment with breast mimicking phantom, coronal slice of 3D volume. (a) Unsubtracted original image. (b) Unsubtracted image after motion correction. (c) Subtraction image prior to motion correction. (d) Subtraction image after motion correction.

without motion, and the post-correction mean ROI in the signal enhancing vial with induced motion. This implies that the motion-correction phase estimates were computed accurately in the contrast-enhanced displaced datasets. Additionally, in a couple of patient datasets we observed substantial enhancement in the breast parenchyma with contrast administration, however motion correction did not result in a mispositioning of this enhancing parenchyma, since it is mostly coaxial with breast fat.

There were a range of results for the clinical breast data, depending on the nature of the motion that might have occurred during the exams. For a typical study we maintained a $32 \mathrm{~cm}$ FOV with $4 \mathrm{~mm}$ thick slices at a $(256 \times 32 \times 32) \mathrm{ma}-$ 
trix size. At these settings the maximum detected slopes, over all patients, corresponded to a displacement of 8,5 , and $3 \mathrm{~mm}$ in $\mathrm{A} / \mathrm{P}, \mathrm{S} / \mathrm{I}$, and $\mathrm{R} / \mathrm{L}$ directions, respectively.

Qualitative visual assessment of the subtraction images showed that in certain cases there was substantial observable motion artifact in the uncorrected datasets, that was successfully removed in the corresponding motion-corrected images. The improvement was most noticeable as a marked reduction in subtraction edge artifact. Low amplitude ghost artifact and blurring in the background was also substantially reduced (Fig. 4). This effect was noticed by the improved clarity of features such as the nipples (arrows). Motion correction also enhanced the visual quality of fine structures such as vessels seen in axial and cross-sectional orientations (Fig. 2). From the plots of mean slope per time point in Fig. 4, we see that the motion correction algorithm has the freedom to individually correct each breast. In this example, greater displacements were detected for the right as compared to the left breast, providing the same overall degree of correction for both breasts.

Quantitative analysis of motion artifact reduction for the clinical cases is summarized in Fig. 5. The mean subtracted artifact ROI intensity over all dynamic timepoints, normalized to the background noise signal intensity, motion corrected vs uncorrected datasets, is plotted in Fig. 5(a). The corresponding plot for the peak artifact ROI is shown in Fig. 5(b). The line of unity represents equivalent artifact in motion corrected and noncorrected datasets. The filled circles in the graphs [Figs. 5(a) and 5(b)] correspond to the peak and mean artifact correction levels for the edge artifact seen in Fig. 4. For this representative artifact we see that there was a roughly $40 \%$ reduction in subtraction edge artifact, resulting in the improved visibility described earlier. We can summarize the results of motion correction in terms of the edge artifact measure as follows:

Motion correction produced a reduction in mean artifact ROI in a number of cases, i.e., there was an improvement in edge artifact suppression. For certain cases there was no significant difference between the mean ROI's for the corrected and uncorrected datasets. This suggests that other sources of phase artifact, such as rotations and distortions, that did not fit the linear three-dimensional translational model, could be present. In none of the cases was the mean artifact ROI greater for the motion corrected dataset compared to the uncorrected dataset. This implies that the correction algorithm did not introduce any additional artifact.

\section{CONCLUSIONS AND DISCUSSION}

Contrast enhanced MRI is developing into a fairly useful tool for the detection and characterization of breast tumors. The potential for breast MRI as a clinical diagnostic tool lies in the ability to achieve volume imaging of both breasts at high spatial resolution, yielding good anatomic detail. In addition contrast enhanced dynamic imaging provides functional information that could assist in tumor characterization. The fidelity of the MRI image data is often limited by artifact sources including motion during the acquisition.

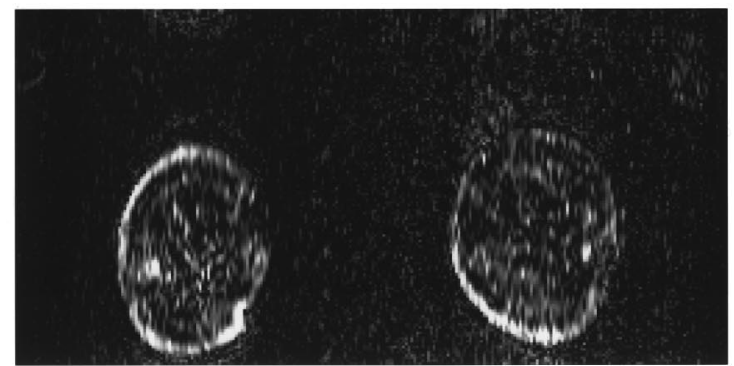

(a)

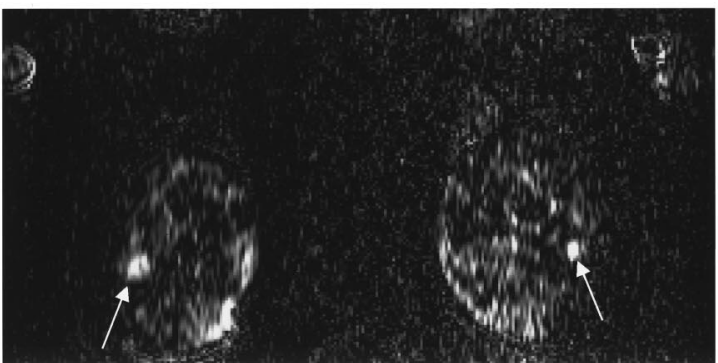

(b)

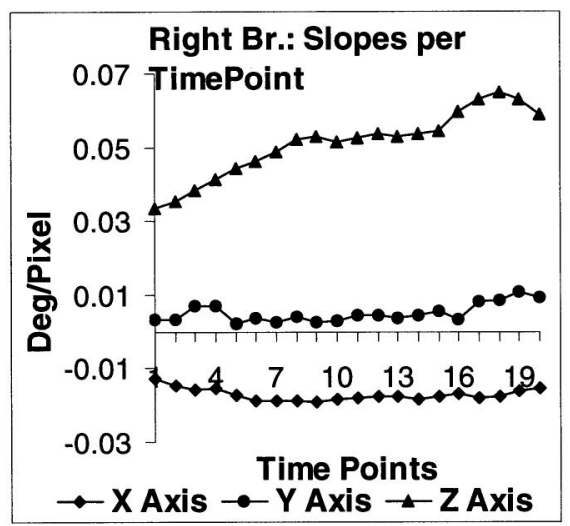

(c)

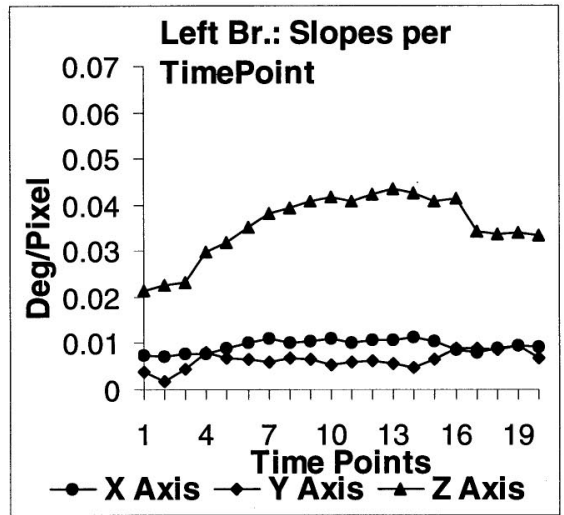

(d)

FIG. 4. Coronal slice of 3D volume acquisition. (a) Subtraction image before motion correction. (b) Subtraction image after motion correction. Note improved clarity of nipples (arrow) and reduced subtraction edge. (c) Mean slopes in $k x, k y$, and $k z$, per time point in left breast. (d) Mean slopes in $k x$, $k y$, and $k z$, per time point in right breast.

In keyhole substitution MRI an effect of linear motion is to introduce phase discontinuities and shifts between the dynamic and reference datasets. These phase deviations manifest as edge artifacts and cause blurring, rather than an ob- 


\section{Motion Corrected VS Non Motion Corrected: Mean} Subtracted ROI Normalized to Background Noise Level

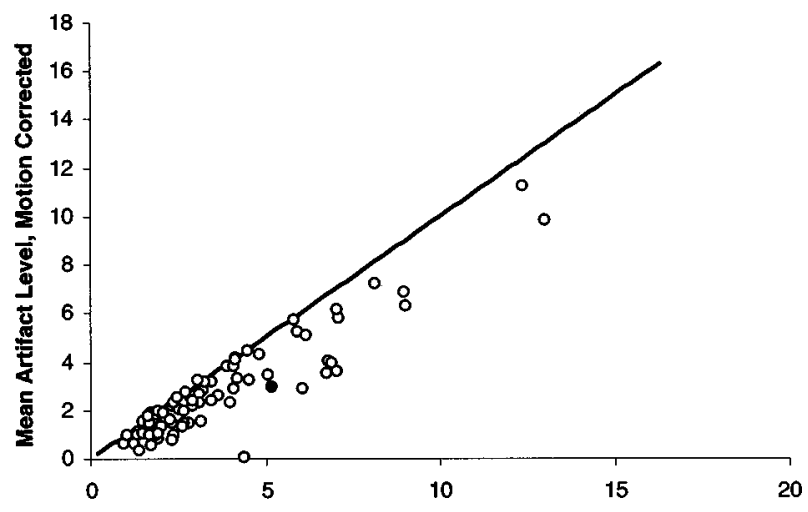

(a)

Mean Artifact Level, Non-Motion Corrected

Motion Corrected VS Non Motion Corrected: Peak Subtracted ROI Normallzed to Background Noise Level

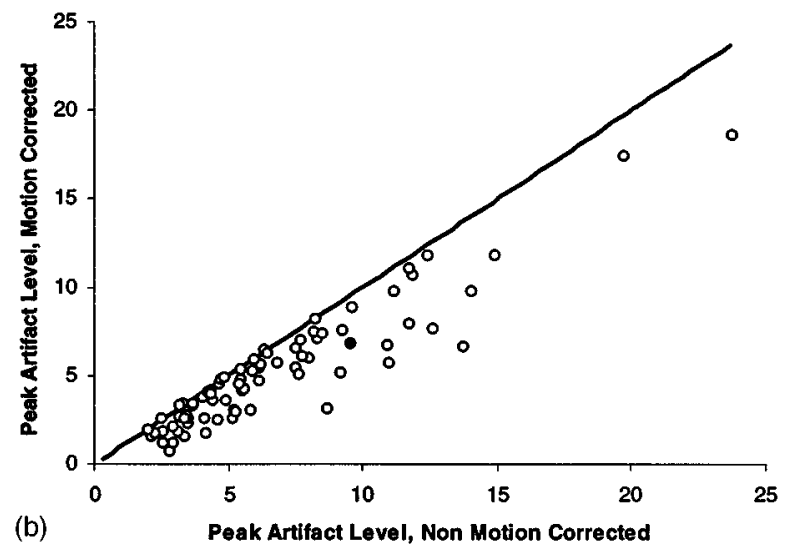

FIG. 5. (a) Comparison of mean artifact level per dynamic series between motion-corrected and uncorrected datasets. (b) Comparison of peak artifact level per dynamic series in motion-corrected and uncorrected datasets.

servable gross displacement, of small lesions in subtraction images that are used for quantitative analysis. This is because the bulk (three fourths) of the spatial information is derived from the peripheral high frequencies in the reference dataset. Figure 6 is an illustration of this effect. We observe that motion produces subtraction edge artifact and some amount of lesion blurring, but retains most of the lesion contrast information. The artifacts can, however, obscure lesions or result in reduced visibility in subtraction images that normally offer the greatest lesion conspicuity. Superficial lesions that lie near high contrast boundaries could remain undetected. Furthermore, identifying lesion ROI's in these artifactually blurred images could lead to inaccurate quantification of contrast uptake characteristics. Thus, effective motion correction algorithms are desirable in contrastenhanced breast MRI.

The scope of this study was limited to gross translational rigid body motion of the breast. The motion correction algorithm assumes no intrakeyhole motion, i.e., motion during the acquisition of a single dynamic block. In this context, calculating averaged phase slope tends to identify gross shifts between the reference and each subsequent dynamic

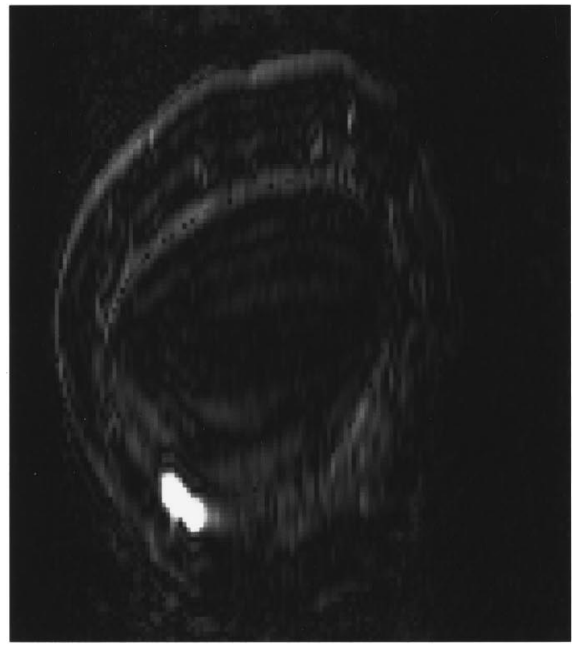

(a)

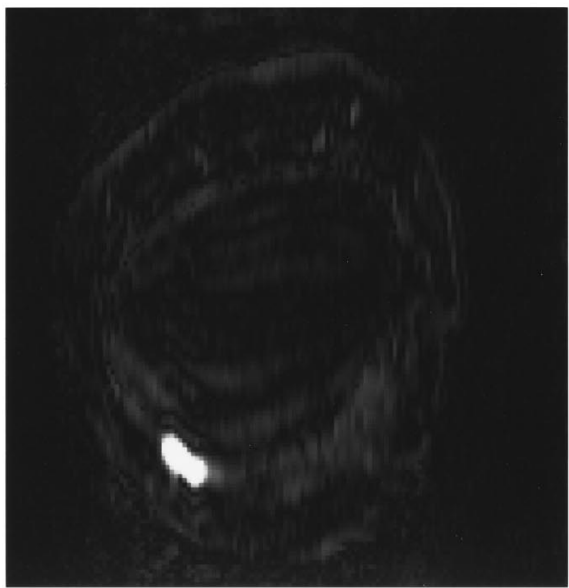

(b)

FIG. 6. Example of subtraction edge due to keyhole reconstruction of motion corrupted dataset, while contrast information is retained. (a) Uncorrected coronal slice, showing malignant lesion and broad subtraction edges. (b) Same slice after correction, showing reduction in edge artifact, while maintaining lesion clarity and contrast. The detected displacements were 1.6, 4.7, and $8.1 \mathrm{~mm}$ along the $x, y$, and $z$ axes.

acquisition. Further, averaging permits the algorithm to be less sensitive to random phase fluctuations that it would be highly susceptible to, if we were to perform a local phase correction per point in $k$-space. However, there are other degrees of motion such as rotation, distortions and respiratory and cardiac motions, that are commonly encountered during the scan. The assumption of no intrakeyhole motion itself does not strictly hold and this combined with heart motion contributes to the low amplitude flutter that is seen on many subtraction images.

An established technique to adaptively correct for both intra and inter-view motion is the navigator echo acquisition. ${ }^{29}$ In this method, an additional echo (NAV) is acquired per phase encoding echo. Since the TR is short ( $\sim 10 \mathrm{~ms}$ ) for the 3D SPGR sequence used for this study, acquisition of the additional navigator echos would substantially increase the scan time, thereby reducing the dynamic temporal sampling rate. Alternatively, a single navigator 
echo can be acquired each, along $k y=0$ and $k z=0$, with $k x=0$ being acquired as part of the dynamic acquisition. This would add negligible time to the 3D acquisition, maintaining the desired high temporal sampling rate. In effect, this approach makes the assumption that a single set of displacements in $x, y$, and $z$ can summarize the motion artifact over the entire volume acquisition. This is similar to the basic assumptions described in this paper. Furthermore, since a displacement in space corresponds to a linear phase roll in $k$-space, the phase estimation technique described in this paper is considered appropriate to detect and correct the expected motions. The averaging over multiple echoes in the dataset serves to provide some degree of noise insensitivity.

In summary, the motion correction algorithm presented in this paper successfully reduces gross translational motion by estimating an averaged linear phase deviation per spatial frequency axis, between the reference and dynamic datasets. Where motion was negligible or could be attributed to other sources, implementation of the algorithm was not detrimental to the original data. If the nature of motion was within the parameters of the model, fairly good correction in terms of improved visualization of structures of interest, reduction in subtraction edges, and suppression of background ghost artifact and blurring was observed.

a) Author to whom correspondence should be addressed. Telephone: (734)936-8865; Fax: (734)-764-2412.

${ }^{1}$ S. H. Heywang et al., "MRI of the breast: histopathological correlation," Eur. J. Radiol. 7, 175-182 (1987).

${ }^{2}$ S. H. Heywang et al., "MR imaging of the breast using gadoliniumDTPA,' J. Comput. Assist. Tomogr. 10, 199-204 (1986).

${ }^{3} \mathrm{~S}$. E. Harms et al., "Mr imaging of the breast with rotating delivery of excitation off resonance: clinical experience with pathologic correlation," Radiology 187, 493-501 (1993).

${ }^{4}$ S. G. Orel et al., "Staging of suspected breast cancer: effect of MR imaging and MR-guided biopsy," Radiology 196, 115-22 (1995).

${ }^{5}$ W. A. Kaiser and E. Zeitler, "MR imaging of the breast: fast imaging sequences with and without Gd-DTPA," Radiology 170, 681-686 (1989).

${ }^{6}$ M. D. Schnall, S. Orel, and L. Muenz, "Analysis of time intensity curved for enhancing breast lesions (abstr)," in Proceedings of the Society of magnetic Resonance in medicine (Berkeley, California Society of Magnetic Resonance in Medicine, 1993), p. 120.

${ }^{7}$ J. P. Stack, O. M. Redmond, M. B. Codd, P. A. Derva, and J. T. Ennis, "Breast disease: tissue characterization with Gd-DTPA enhancement profiles,' Radiology 174, 491-494 (1990).

${ }^{8}$ F. W. Flickinger, J. D. Allison, R. M. Sherry, and J. C. Wright, "Differentiation of benign from malignant breast masses by time-intensity evaluation of contrast enhanced MRI," Magn. Reson. Imaging 11, 617-620 (1993).

${ }^{9}$ N. Seidner, J. P. Semple, W. R. Welch, and J. Folkman, "Tumor angiogenesis and metastasis: correlation in invasive breast carcinoma," $\mathrm{N}$. Engl. J. Med. 324, 1-8 (1991).
${ }^{10}$ J. Folkman, "What is the evidence that tumors are angiogensis dependent?," J. Natl. Cancer Inst. 82, 4-6 (1990).

${ }^{11}$ A. L. Harris and E. Horak, "Growth factors and angiogenesis in breast cancer. Recent results," Cancer Res. 127, 35-41 (1993).

${ }^{12}$ H. M. Jensen, J. Chen, M. R. DeVault, and A. E. Lewis, "Angiogenesis induced by "normal" human breast tissue: a probable marker for precancer," Science 218, 293-295 (1982).

${ }^{13}$ S. H. Heywang-Korbrunner, "Contrast-enhanced magnetic resonance imaging of the breast," Invest. Radiol. 29, 94-104 (1994).

${ }^{14}$ T. A. Spraggins, E. S. de Paredes, G. A. De Angelis, and F. Thiele, "Three dimensional keyhole imaging: application of dynamic contrast enhanced MRI of breast lesions (abstr)," in Proceedings of the Society of Magnetic Resonance Imaging in Medicine (Berkeley, California Society of Magnetic Resonance in Medicine, 1993), p. 118.

${ }^{15}$ S. E. Harms, D. P. Flamig, and K. L. Hesley, "Fat-suppressed threedimensional MR imaging of the breast," RadioGraphics 13, 247-267 (1993).

${ }^{16}$ Z. P. Liang and P. C. Lauterbur, "An efficient method for dynamic magnetic resonance imaging," IEEE Trans. Med. Imaging 10(4), 677-686 (1994).

${ }^{17} \mathrm{~V}$. Rasche et al., "Helical scan for time resolved MRI at $0.5 T$ (abstr)," in Proceeding of the Society of Magnetic Resonance in Medicine (Berkeley, California, Society of Magnetic Resonance in Medicine, 1993), p. 479.

${ }^{18} \mathrm{~J}$. W. Black et al., "Magnetic Resonance Imaging of Breast Disease Using a Dynamic Spiral Sequence,' in Proceedings of the International Society of Magnetic Resonance in Medicine (Berkeley, California, Society of Magnetic Resonance in Medicine, 1996), p. 749.

${ }^{19}$ J. J. Van Vaals et al., "Keyhole method of accelerating imaging of contrast agent uptake,' Magn. Reson. Imaging 3, 671-675 (1993).

${ }^{20} \mathrm{D}$. B. Plewes et al., "Errors in quantitative dynamic three-dimensional keyhole MR imaging of the breast," Magn. Reson. Imaging 5(3), 361-4 (1995).

${ }^{21}$ T. L. Chenevert and J. G. Pipe "Dynamic 3D imaging at high temporal resolution via reduced $k$-space sampling (abstr)," in Proceeding of the Society of Magnetic Resonance in Medicine (Berkeley, California, Society of Magnetic Resonance in Medicine, 1993), p. 1262.

${ }^{22}$ T. L. Chenevert et al., "Dynamic three-dimensional imaging with partial $K$-space sampling: Initial application for Gadolinium enhanced rate characterization of breast lesions," Radiology 196(1), 135-42 (1995).

${ }^{23}$ H. W. Korin, F. Farzaneh, R. C. Wright, and S. J. Riederer, "Compensation for the effects of linear motion in MR imaging," Magn. Reson. Med. 129(1), 99-113 (1989).

${ }^{24}$ V. J. Wedeen, R. E. Wendt, and M. Jerosch-Herold, "'Motion phase artifacts in Fourier transform MRI," Magn. Reson. Med. 11(1), 114-120 (1989).

${ }^{25}$ E. M. Haacke and J. L. Patric, "Reducing motion artifacts in twodimensional Fourier Transform imaging,' Magn. Reson. Med. 4, 359376 (1986).

${ }^{26} \mathrm{M}$. Hedley and H. Yan, "Motion artifact suppression: A review of postprocessing techniques,' Magn. Reson. Imaging 10, 627-635 (1992).

${ }^{27} \mathrm{M}$. Hedley, H. Yan, and D. Rosenfeld "Motion artifact correction in MRI using generalized projections,' IEEE Trans. Med. Imaging 10, 40-46 (1991).

${ }^{28}$ R. N. Bracewell, The Fourier Transform and its Applications (McGrawHill, New York, 1986), pp. 104-105.

${ }^{29}$ J. P. Felmlee, R. L. Ehman, D. I. Riederer, and H. W. Korin, "Adaptive Motion Compensation in MRI: Accuracy of motion measurements," Magn. Reson. Med. 18, 207-213 (1991). 